\title{
Emergence of pediatric hospital medicine: A novel subspecialty in the Middle East
}

\author{
Jaidev Nath, Yousra Ghoweba \\ Pediatric Department, Al Jalila Children's Hospital, Dubai, UAE
}

Received: 2021-06-15.

Accepted: 2021-09-05

This work is licensed under a Creative Commons Attribution 4.0 International License

\begin{abstract}
In this article, we give an overview of the hospital medicine model, which has recently been adopted by multiple healthcare systems. We will focus particularly on how pediatric facilities can transition to the pediatric hospital medicine model and reap its benefits.
\end{abstract}

Key words: pediatrics, hospital medicine, transition

J Clin Med Kaz 2021; 18(5):4-5

Corresponding author:

Jaidev Nath.

E-mail: jaidev.nath@ajch.ae.

ORCID: 0000-0002-0913-1686

\section{Hospital Medicine Model}

The term 'hospitalist' was coined in 1996 [1]. It described physicians whose primary area of expertise was managing the care of hospitalized patients. In the United States, the number of hospitalists began to rise in the late 1990s. The main driver behind this growth was an effort to achieve greater efficiency by reducing lengths of stay and decreasing care-related costs [2]. In the last two decades, hospital medicine has continued to grow at a rapid pace in the United States with some expansion internationally [3].

One of the major advantages of the Hospital Medicine model is that it allows for the growth of expertise in the inpatient setting for hospitalists, and in the outpatient setting for primary care physicians. Additionally, having a dedicated hospitalist physician improves care for higher acuity patients with multiple co-morbidities who require full time physician presence in the hospital [4]. Some of the challenges faced by outpatient pediatricians are the increasing complexity of patients, seeing more patients in less time while thoroughly documenting each encounter. Adoption of a hospital medicine model allows the outpatient pediatrician the time and bandwidth to overcome these challenges. Managing complex patients (eg. failure to thrive) at an inpatient setting facilitates a multidisciplinary approach due to easier access to different subspecialties and longer duration of patient encounter compared to the outpatient setting. One of the perceived disadvantages of this model is the loss of continuity of care by the primary physician when a patient is hospitalized. However, the rapid growth of hospital medicine suggests that the benefits of this model outweigh its weaknesses.

\section{Pediatric hospital medicine model}

Paralleling its adult counterpart, pediatric hospital medicine has had tremendous growth over the last two decades [5]. It is currently one of the fastest growing pediatric subspecialties in the United States [5]. This is driven by the rising complexity of pediatric patient population and need for dedicated teams and experts to manage the inpatient setting. Acknowledging the growth and impact of pediatric hospital medicine, it was officially recognized as a sub-specialty by the American Board of Medical Subspecialties in 2016 and the first certifying examination was administered in 2019. 
Outside of North America, a traditional blended model of general pediatric care continues to dominate in most parts of the world, including the Middle East. In this blended model, hospitalized patients are managed by a team of junior physicians and residents who are led by a pediatric consultant. Following ward rounds, the consultant often proceeds to see patients in the clinics, while junior doctors continue to provide the ongoing hands on care for hospitalized patients.

\section{Transitioning to the pediatric hospital medicine model}

Transitioning to a pediatric hospital medicine model in the Middle East might be challenging as many pediatricians will be hesitant to let go of a large piece of their practice - whether it be inpatient or outpatient care. However, this can be mitigated by offering incentives to physicians who agree to make this shift and ensuring that the wards will be adequately staffed to avoid physician burnout. More importantly, it will be crucial to provide continuous medical education to inpatient physicians to enhance and update their clinical knowledge and skills.
After hospitals transition to this model by reallocating their staff either to the inpatient or outpatient setting, they should aim in the future to recruit only accredited pediatric hospitalists when staffing their inpatient units. This will support the growth of this model moving forward.

One example of a hospital that successfully transitioned to the pediatric hospital medicine model in the region is Al Jalila Children's Specialty Hospital in the United Arab Emirates. They can be used as a benchmark by hospitals that aspire to make such a transition. The implementation of this model should be strongly considered by healthcare authorities in the region, as it will to contribute to enhancing patient care and improving outcomes along various touch points within the healthcare system.

Disclosures: There is no conflict of interest for all authors.

Acknowledgements: None.

Funding: None.

\section{References}

1. Wachter RM, Goldman L: The emerging role of 'hospitalists' in the American health care system. N Engl J Med. 1996; 335:514-7. DOI: 10.1056/NEJM199608153350713

2. Messler J, Whitcomb WF: A History of the Hospitalist Movement. Obstet Gynecol Clin North Am. 2015; 42:419-432. DOI: 10.1016/j. ogc.2015.05.002

3. Kisuule F, Howell E: Hospital medicine beyond the United States. Int J Gen Med. 2018; 11:65-71. DOI: 10.2147/IJGM.S151275

4. Auerbach AD, Wachter RM, Katz P, Showstack J, Baron RB, Goldman L: Implementation of a voluntary hospitalist service at a community teaching hospital: improved clinical efficiency and patient outcomes. Ann Intern Med. 2002; 137:859-65. DOI: 10.7326/0003-4819137-11-200212030-00006

5. Rauch DA, Lye PS, Carlson D, et al.: Pediatric hospital medicine: a strategic planning roundtable to chart the future. J Hosp Med. 2012; 7:329-34. DOI: 10.7326/0003-4819-137-11-200212030-00006 